\title{
Study on the mechanism of the Modified Ginseng-Schisandra Decoction (MGSD) in the treatment of recurrent respiratory tract infection (RRTI) based on network pharmacology
}

\author{
Qing-Ke Cui ${ }^{1}$, $\mathrm{Hua} \mathrm{Li}^{1}{ }^{1}, \mathrm{Zhan}_{\mathrm{Li}^{1}}, \mathrm{Jie} \mathrm{Li}^{1}$, Liqi Song ${ }^{2}$ \\ ${ }^{1}$ Department of Chinese Medicine, Shanghai Children's Hospital, Shanghai Jiao Tong University, Shanghai, China; ${ }^{2}$ Jilin Province Jilin Hospital of \\ Integrated Chinese and Western Medicine, Jilin, China \\ Contributions: (I) Conception and design: QK Cui; (II) Administrative support: H Li; (III) Provision of study materials or patients: Z Li; (IV) \\ Collection and assembly of data: J Li; (V) Data analysis and interpretation: L Song; (VI) Manuscript writing: All authors; (VII) Final approval of \\ manuscript: All authors. \\ Correspondence to: Hua Li. Chief Physician, Master Tutor. Department of Chinese Medicine, Shanghai Children’s Hospital, Shanghai Jiao Tong \\ University, Shanghai, China. Email: drlihua502@126.com.
}

Background: The aim of this study was to investigate the mechanism of Modified Ginseng-Schisandra Decoction in the treatment of recurrent respiratory tract infection (RRTI) using network pharmacology.

Methods: To screen the active ingredients of A Modified Ginseng-Schisandra Decoction, TCMSP, TCMID, Batman-TCM and PubChem database were applied. To predict the targets of active ingredients on RRTI, TCMSP, Pubmed, OMIM, Drug Bank, GAD and TTD database were used. The compoundstherapeutic target network was constructed with Cytoscape 3.7.2 software. The STRING database was used to construct a protein-protein interaction (PPI) network, and Kyoto Encyclopedia of Genes and Genomes (KEGG) pathway enrichment analysis was used to identify potential signal pathways.

Results: The 3 main active ingredients of Modified Ginseng-Schisandra Decoction obtained by screening were quercetin, kaempferol, and isoflavone; the main therapeutic targets were PTGS2, ESR1, AR, PPARG, NOS2, and others. Based on the PPI network, we found that the targets of Modified Ginseng-Schisandra Decoction were significantly enriched in (FDR <0.01) cancer pathway, tumor necrosis factor (TNF) signaling pathway, hypoxia-inducible factor (HIF-1) signaling pathway, and others.

Conclusions: Modified Ginseng-Schisandra Decoction can treat RRTI primarily through acting in the signal transduction of some key nodes of cancer pathway and TNF pathway. It exerts a direct or indirect influence on multiple signaling pathways, and has the characteristics of multicomponent, multitarget, and multichannel action.

Keywords: Modified Ginseng-Schisandra Decoction; recurrent respiratory tract infection (RRTI); network pharmacology; signal pathway; molecular mechanisms

Submitted May 18, 2021. Accepted for publication Jun 17, 2021.

doi: $10.21037 / \mathrm{tp}-21-240$

View this article at: https://dx.doi.org/10.21037/tp-21-240

^ ORCID: Qing-Ke Cui, 0000-0002-0483-8383; Hua Li, 0000-0002-1193-0452. 


\section{Introduction}

Recurrent respiratory tract infection (RRTI) is a common and frequently occurring disease in children. It refers to the frequent occurrence of upper and lower respiratory tract infections within 1 year that exceed the normal range $(1,2)$. According to the location of infection, RRTI can be divided into recurrent upper respiratory tract infection and recurrent lower respiratory tract infection (bronchitis and pneumonia) (1). According to statistics, the incidence of RRTI among children under the age of 1 in developed countries is as high as $25 \%$, while the incidence in children between 1 and 4 years of age is 18\% (3). In developing countries, the annual number of child deaths from RRTI is as high as 2 million (mainly pneumonia) (4). Relevant investigations show that this disease accounts for about 10 $20 \%$ of respiratory diseases in China, with $70-80 \%$ of these patients being under 5 years old (5). As conditions in the environment change, the incidence of RRTI is increasing (6). Compared with lower respiratory tract infection, repeated upper respiratory tract infection is more common (7). RRTI is characterized by a long duration and a high likelihood of multiple recurrences. Children with severe disease can have several episodes per month. If the disease is not treated in timely and effective fashion, it can develop into chronic disease, with asthma, nephritis, myocarditis being common complications (8) that pose a considerable threat to the life and health of children (7). Traditional Chinese medicine (TCM) has attracted much attention in the prevention and treatment of RRTI in recent years.

RRTI can be classified as "body deficiency cold", "cough and asthma", "long cough", "deficiency syndrome" and "spontaneous sweating" in the discourse of traditional Chinese medicine (9). The key pathogenesis lies in "deficiency" (10), and clinical treatment is based on stage treatment and syndrome differentiation.

Modified Ginseng-Schisandra Decoction is often used in the treatment of RRTI ("lung and spleen deficiency syndrome"), Ginseng-Schisandra decoction comes from a book named "Youyou Jicheng" written by Fuzheng Chen during the Qing dynasty. The original record is as follows: "The cough lasts for a long time, and there is no other evidence. It is due to lung deficiency. Only the spleen should be supplemented. Modified Ginseng-Schisandra Decoction. It's also a magical prescription to treat long-term cough and deficiency of qi and weak qi, white face, and white lips." The original prescription consists of ginseng, Atractylodes macrocephala Koidz., Poria cocos, Schisandra, Ophiopogon japonicus, Radix Glycyrrhizae Preparate, ginger, and jujube. Later doctors often used this prescription to treat cough due to deficiency of lung and spleen qi. Based on clinical experience, we use Modified Ginseng-Schisandra Decoction to treat spleen deficiency syndrome after RRTI. The basic prescription is composed of Radix astragali (6 g), Codonopsis pilosula (6 g), Atractylodes macrocephala Koidz. (6 g), Poria cocos (9 g), Schisandra (3 g), Ophiopogon japonicus (6 g), oyster (15 g), Radix glycyrrbizae Preparate (3 g), and Saposhnikovia divaricata $(10 \mathrm{~g})$, with the ingredients being added or subtracted based on clinical symptoms. As for Children with RRTI, Modified Ginseng-Schisandra Decoction is used according to the following rules: 1 to 2 years old: 2 times/day, 1/3 doses/time; 3 to 6 years old; 2 times/day, 0.5 doses/time; 6 years old and above, 2 times/day, 1 dose/time. The treatment course lasted 4 weeks. Preliminary clinical studies have found that the Modified Ginseng-Schisandra Decoction can reduce TCM syndrome scores, and promote white blood cell (WBC), hemoglobin ( $\mathrm{Hb}), \mathrm{C}$-reactive protein (CRP), and eosinophil (EOS) and other indicators to return to normal levels, with the clinical efficacy being superior to that of single-use Bacillus subtilis dual-live bacteria particles for treatment $(11,12)$.

TCM prescriptions typically have multiple components, multiple targets, and multiple pathways. This study used network pharmacology to examine the potential pharmacological effects and molecular mechanisms of Modified Ginseng-Schisandra Decoction on the treatment of RRI, so as to lay a theoretical foundation for its treatment of RRTI. We present the following article in accordance with the MDAR reporting checklist (available at https:// dx.doi.org/10.21037/tp-21-240).

\section{Methods}

\section{Research design}

The general experimental design for this pharmacology study of Modified Ginseng-Schisandra Decoction in the treatment of RRTI included the following principal steps: the screening of active ingredients of Modified GinsengSchisandra Decoction, the establishment of a target database of active ingredients related to RRTI, and the establishment of the Modified Ginseng-Schisandra Decoction-Active compounds-therapeutic target network with subsequent KEGG enrichment analysis. The study was conducted in accordance with the Declaration of Helsinki (as revised in 2013). 


\section{Database establishment}

Screening the main active ingredients of the Modified Ginseng-Schisandra Decoction

We searched the Traditional Chinese Medicine Systems Pharmacology Database and Analysis Platform (TCMSP), Traditional Chinese Medicines Integrated Database (TCMID), and Batman-TCM (http://bionet.ncpsb. org/batman-tcm/) databases to find the main chemical components of Radix astragali, Codonopsis pilosula, Atractylodes macrocephala Koidz., Poria cocos, Schisandra, Ophiopogon japonicus, oyster, Radix glycyrrhizae Preparate, and Saposhnikovia divaricata, and used the PubChem database (https://pubchem.ncbi.nlm.nih.gov/) to check their compound names and molecular structures. With oral bioavailability $(\mathrm{OB}) \geq 30 \%$ and drug-likeness (DL) $\geq 0.18$ as screening conditions, the compounds were screened and the active compound targets were obtained.

\section{Prediction of targets of active ingredients in Modified Ginseng-Schisandra Decoction}

The TCMSP database revealed the targets corresponding to the active ingredients of the Modified Ginseng-Schisandra Decoction. The targets from different sources were entered into UniProt (http://www.uniprot.org), PubMed, and other databases with the search limited to the human species. In this way, the gene symbols corresponding to the targets were acquired for subsequent network pharmacological analysis.

\section{Target mining of RRTIs}

The keyword phrase "Recurrent respiratory tract infection" was used to search the Online Mendelian Inheritance in Man (OMIM) database (https://www.omim.org), Drug Bank (http://www.drugbank.ca/), Gene Association Database (GAD; http://geneticassociation-db.nih.gov/), and Therapeutic Targets Database (TTD http://db.idrblab.net/ ttd/) for all RRTI targets.

\section{Construction and visualization of Modified Ginseng- Schisandra Decoction network diagram}

Construction and visualization of Modified GinsengSchisandra decoction-Active compounds-therapeutic target

Drug-disease targets (DDTs) were obtained from identifying the intersection of Modified Ginseng-Schisandra Decoction target and the RRTI target with Venn (http:// bioinformatics.psb.ugent.be/webtools/Venn/. The Modified
Ginseng-Schisandra Decoction-Active compoundstherapeutic target network was constructed with Cytoscape 3.7.2 software, and topology analysis and visualization were performed. The Network Analyzer tool in the software was used to calculate the degree value to evaluate the importance of the components and targets in the network.

\section{Protein-protein interaction (PPI) network data}

The Modified Ginseng-Schisandra decoction PPI network was constructed using the Search Tool for the Retrieval of Interacting Genes/Proteins (STRING) database (https:// string-db.org/). The conditions were set to "minimum required interaction score $=0.9$ " and "hide disconnected nodes in the network".

\section{Kyoto Encyclopedia of Genes and Genomes enrichment analysis}

To further elucidate the different scientific connotations of Modified Ginseng-Schisandra decoction from a biological perspective, we used the KOBAS online server (http:// kobas.cbi.pku. edu.cn/kobas3/?T=1) with the species set to human to conduct Kyoto Encyclopedia of Genes and Genomes (KEGG) enrichment analysis and Gene Ontology (GO) function enrichment analysis for the DDTs of the Modified Ginseng-Schisandra Decoction. This was followed by visualization using the KEGG Mapper tool (https:// www.genome.jp/kegg/mapper.html) to analyze the specific role of related targets in important signaling pathways and to obtain a schematic diagram of Modified GinsengSchisandra Decoction regulatory signaling pathways (13).

\section{Statistical analysis}

The relative data were recorded with EXCEL. In KEGG pathway enrichment analysis, the pathways with $\mathrm{P}<0.05$ were considered statistical different and selected.

\section{Results}

\section{Identifying the common targets of the Modified Ginseng- Schisandra Decoction and RRTI}

A total of 156 active compounds of the Modified GinsengSchisandra Decoction (excluding duplicate compounds) were found by searching the three databases. Among them, $15,16,4,6,7,3,14,88$, and 16 corresponding compounds of Radix astragali, Codonopsis pilosula, Atractylodes macrocephala 


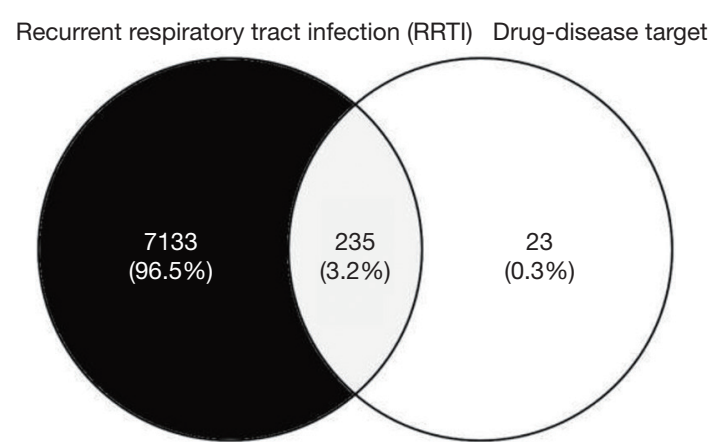

Figure 1 Drug-disease target Venn diagram of Modified GinsengSchisandra Decoction.

Koidz., Poria cocos, Schisandra, Ophiopogon japonicus, oyster, Radix glycyrrbizae Preparate, and Saposhnikovia divaricata were found, respectively.

After Uniprot corrected all target names, the number of potential targets of Modified Ginseng-Schisandra Decoction was 258. Using the GeneCards database, we searched and downloaded all 7,168 target genes related to RRTI. The integration of the potential targets of Modified Ginseng-Schisandra Decoction active chemical components with the targets of RRTI, resulted in 235 DDTs of the Modified Ginseng-Schisandra Decoction (Figure 1).

\section{Analysis and visualization of the DDT network diagram based on the Modified Ginseng-Schisandra decoction}

Cytoscape software was used to construct the DDT network graph. It was composed of 415 nodes and 2,130 edges. In Figure 2, the green represents the prescription drugs of Modified Ginseng-Schisandra Decoction [9], the red represents the active compounds of the Modified GinsengSchisandra Decoction [156], and the blue represents the DDT of Modified Ginseng-Schisandra Decoction [235].

The top 10 therapeutic ingredients with degree value were quercetin, kaempferol, isoflavones (among others) (degrees of 268, 102, and 62, respectively; see Table 1 for details). The top 10 treatment targets by degree were PTGS2, ESR1, AR, PPARG, NOS2 (among others) (degrees of 108, 93, 88, 81, and 74, respectively; see Table 2 for details).

\section{Analysis of the DDT PPI network of the modified Ginseng-Schisandra Decoction}

A total of 235 DDTs of the Modified Ginseng-Schisandra
Decoction were imported into the STRING database (https://string-db.org/). With the filter condition set to "Homo sapiens" and the conditions set to "minimum required interaction score $=0.9$ " and "hide disconnected nodes" in the network", 216 target proteins were obtained. According to the degree value of the network topology index, a bar graph of the top 30 related protein nodes was drawn (Figure 3).

\section{Enrichment analysis of the KEGG signal pathway of the core PPI}

KEGG pathway analysis was performed on 216 target proteins in the PPI network with the condition of a false discovery rate (FDR) set as $<0.01$, and the top 30 signal pathways were visualized according to the FDR value. The specific results are shown in Figure 4. According to the correlation between the size of FDR, the size of enrichment factors, and RRTI, it was found that that important signal pathways included cancer signal pathway, tumor necrosis factor (TNF) signal pathway, hypoxia-inducible factor (HIF-1) signal pathway, among others. The KEGG Mapper tool was used to analyze the specific role of the Modified Ginseng-Schisandra Decoction in the cancer pathway, TNF signaling pathway, and HIF-1 signaling pathway, and the model diagrams of Modified Ginseng-Schisandra Decoction regulating the above signal pathways were constructed (Figures 5,6).

\section{Discussion}

The Modified Ginseng-Schisandra Decoction is composed of Radix astragali, Codonopsis pilosula, Atractylodes macrocephala Koidz., Poria cocos, Schisandra, Opbiopogon japonicus, oyster, Radix glycyrrbizae Preparate, Saposhnikovia divaricata, and is derived from the combination of Chinese classical prescriptions including the Ginseng-Schisandra Decoction, Yupingfeng Powder, Sijunzi Decoction, and Shengmaiyin. Chinese medicine believes that children are pure Yang bodies, and if ginseng is not used properly, it is easy to develop Yang heat and "excessive internal heat" (14). Codonopsis pilosula is sweet and flat, and is more suitable for children. Therefore, the original ginseng is exchanged for Codonopsis. Codonopsis pilosula and Radix astragali are principal drugs, which correspond to the spleen and lung meridian. Codonopsis pilosula has the effects of tonifying the middleJiao and Qi, invigorating the spleen and lungs, invigorating the blood and activating fluid, and eliminating dysphoria. 


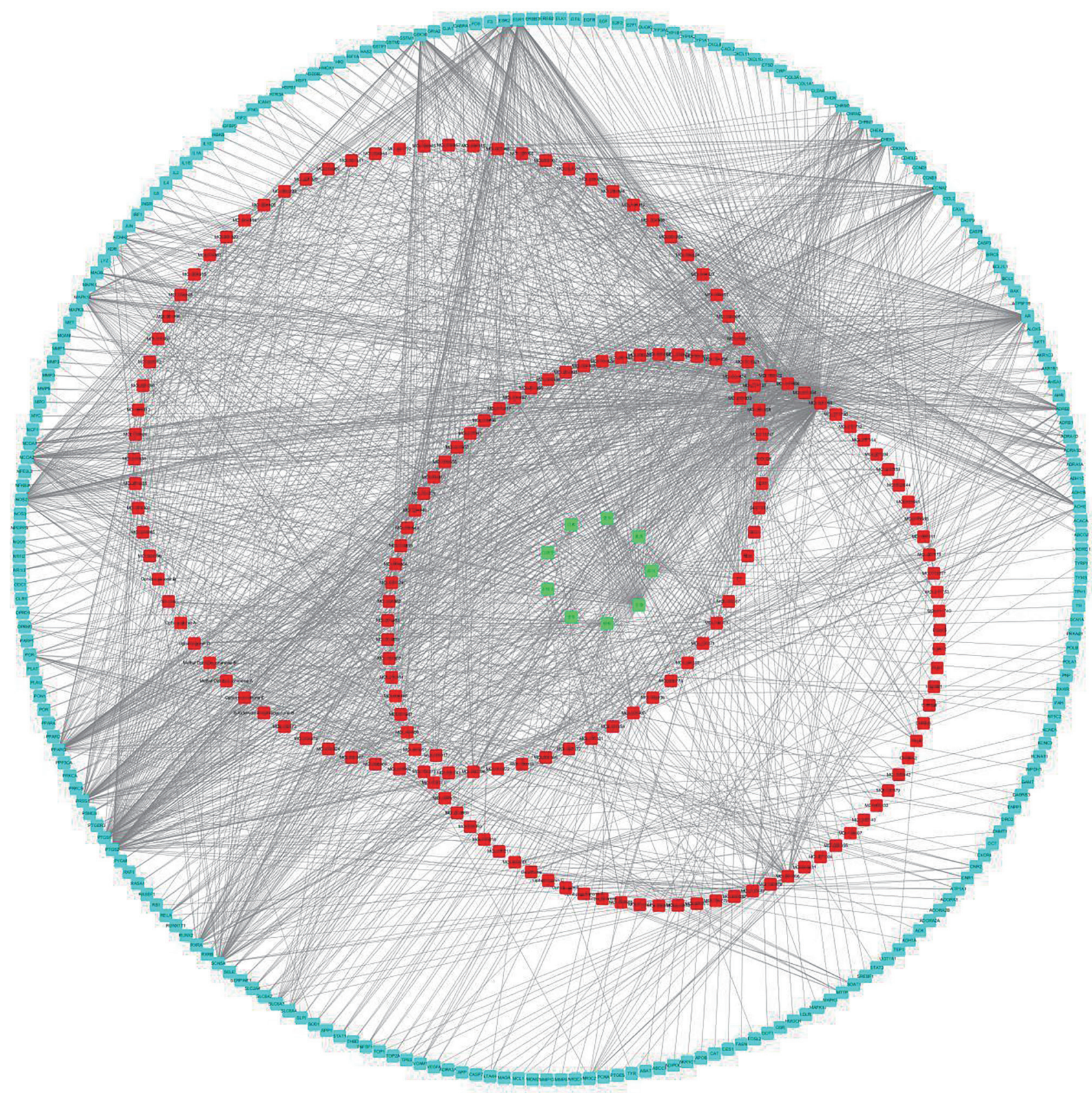

Figure 2 Drug-disease target network diagram of the Modified Ginseng-Schisandra Decoction.

It can enhance immune function, regulate the intestinal tract, reduce the absorption of inflammatory mediators and toxins, and has a good blood-replenishing effect (15). Radix astragali is good at entering the spleen and stomach. It is an important medicine for tonifying the middle-Jiao and Qi. It can tonify the lung and spleen, replenish qi for strengthening superficies. Atractylodes macrocephala Koidz combined with Poria cocos can strengthen the spleen, dry dampness, and replenish qi; Schisandra with Ophiopogon japonicus has the functions of nourishing Yin and moistening lung, benefiting stomach and promoting body fluid, clearing away the heart fire and eliminating irritability; Saposhnikovia divaricata has the functions of dispelling wind, relieving the superficies, and eliminating dampness. According to 
Table 1 Information of the top 10 active ingredients by degree value of Modified Ginseng-Schisandra Decoction

\begin{tabular}{llcc}
\hline Mol ID & Molecule name & OB (\%) & DL \\
\hline MOL000442 & $\begin{array}{l}\text { 1,7-Dihydroxy-3,9-dimethoxy } \\
\text { pterocarpene }\end{array}$ & 39.05 & 0.48 \\
MOL000098 & Quercetin & 46.43 & 0.28 \\
MOL003896 & 7-Methoxy-2-methyl isoflavone & 42.56 & 0.2 \\
MOL000392 & Formononetin & 69.67 & 0.21 \\
MOL000354 & Isorhamnetin & 49.6 & 0.31 \\
MOL000006 & Luteolin & 36.16 & 0.25 \\
MOL000296 & Hederagenin & 36.91 & 0.75 \\
MOL004328 & Naringenin & 59.29 & 0.21 \\
MOL000417 & Calycosin & 47.75 & 0.24 \\
MOL000378 & 7-O-methylisomucronulatol & 74.69 & 0.3 \\
\hline
\end{tabular}

OB, oral bioavailability; DL, drug-likeness.
Table 2 Top 10 drug-disease targets with degree of the Modified Ginseng-Schisandra Decoction

\begin{tabular}{lll}
\hline $\begin{array}{l}\text { Uniprot } \\
\text { ID }\end{array}$ & Target & Degree \\
\hline P35354 & Prostaglandin G/H synthase 2, PTGS2 & 108 \\
Q9SAD4 & Ethylene-responsive transcription factor, ESR1 & 93 \\
P10275 & Androgen receptor, AR & 88 \\
P37231 & Peroxisome proliferator-activated receptor & 81 \\
& gamma, PPARG & \\
P35228 & Nitric oxide synthase, NOS2 & 74 \\
P07477 & Trypsin-1, PRSS1 & 73 \\
Q15596 & Nuclear receptor coactivator 2, NCOA2 & 69 \\
P49841 & Glycogen synthase kinase-3 beta, GSK3B & 66 \\
Q9FYK5 & Ethylene-responsive transcription factor, ESR2 & 64 \\
P23219 & Prostaglandin G/H synthase 1, PTGS1 & 63 \\
\hline
\end{tabular}

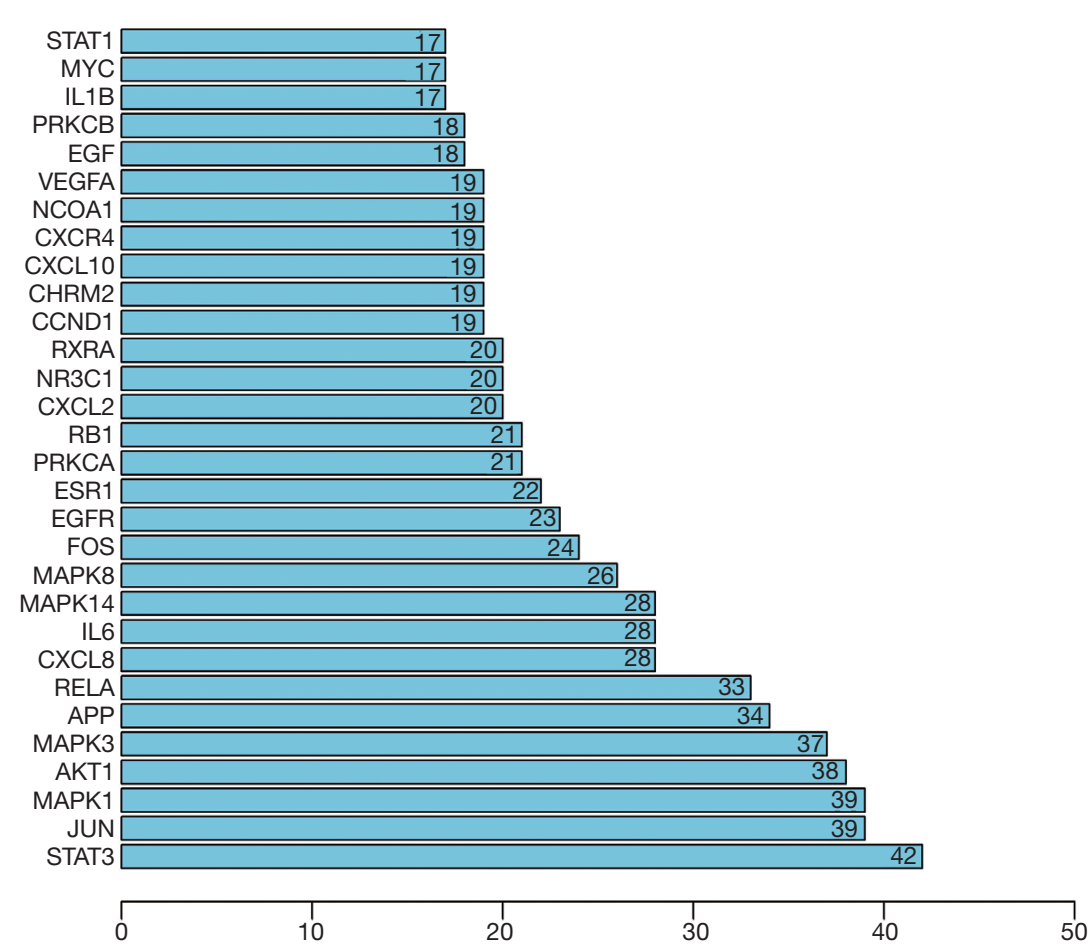

Figure 3 Degree bar chart of the PPI network target protein of the Modified Ginseng-Schisandra Decoction. PPI, protein-protein interaction network. 


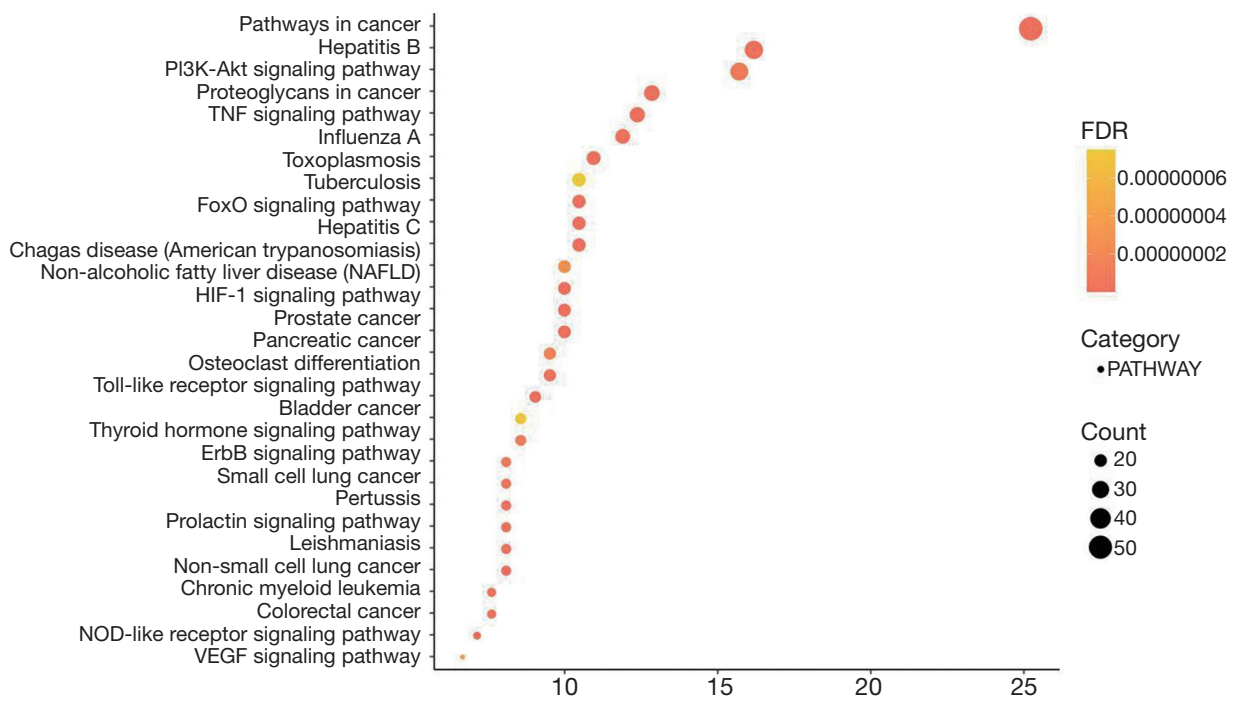

Figure 4 The main signaling pathways involved and mediated by the Modified Ginseng-Schisandra Decoction (top 30).

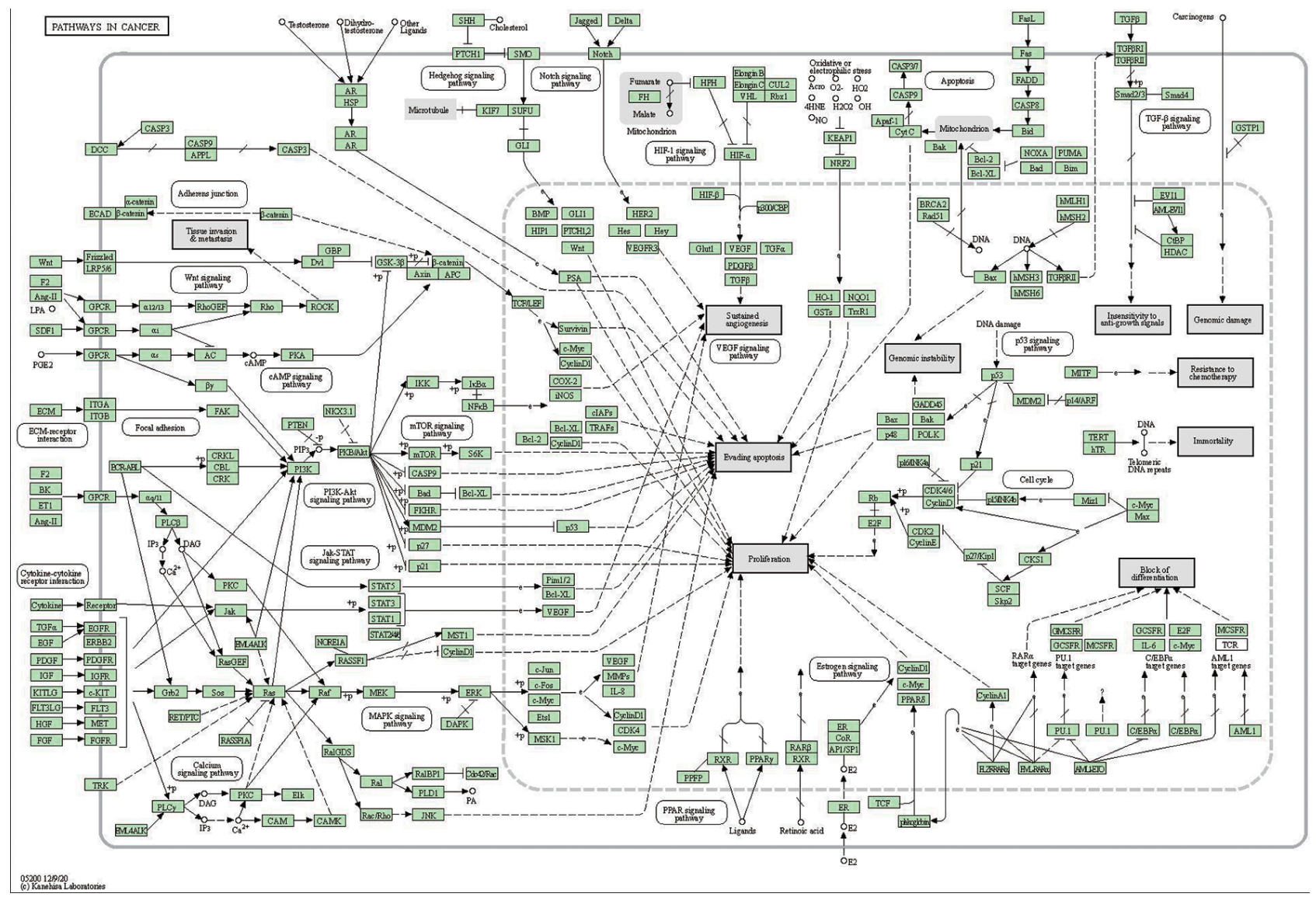

Figure 5 Cancer signaling pathway (Obtained from KEGG database) (13). 

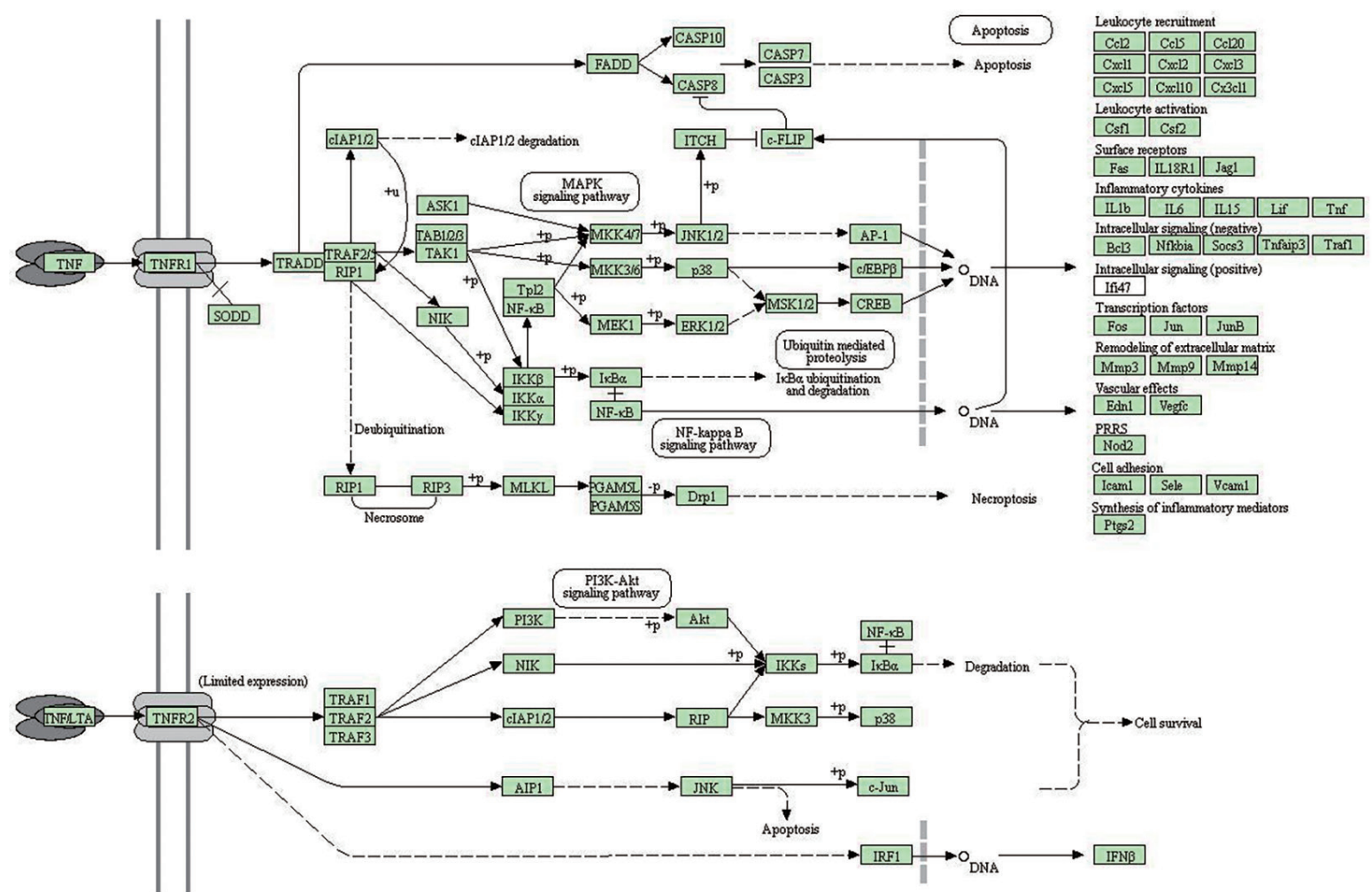

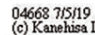

Figure 6 Tumor necrosis factor signaling pathway (Obtained from KEGG database) (13).

modern pharmacological research, Saposhnikovia divaricata has antipyretic, analgesic, anti-inflammatory, antibacterial, antiallergic, and regulating immune function effects (16). Traditional Chinese medicine believes that oysters have the functions of submerging Yang and replenishing Yin, reconciling Yin and Yang, and the combination of Yin and Yang means that "Righteousness is stored in the body, and evil cannot be dried." Modern research has found that oysters' chemical composition has calcium carbonate, iron, zinc, and other trace elements, and is rich in protein zinc and has a wide range of pharmacological activities (17), which can enhance the body's defense against nonspecific stimuli. Radix glycyrrbizae Preparate can tonify the spleen, replenish qi and harmonize various medicines. The Modified Ginseng-Schisandra Decoction has the effects of nourishing the spleen and qi, nourishing Yin, and moisturizing the lungs.

The DDT network diagram of the Modified Ginseng Decoction in this study shows that, from the point of view of a single medicine, Radix glycyrrbizae Preparate can have up to
88 compounds, Codonopsis pilosula and Saposhnikovia divaricata have 16, and Radix astragali has 15 . This suggests that Radix glycyrrbizae Preparate, Codonopsis pilosula, Radix astragali, and Saposhnikovia divaricata may play a more important role in the Modified Ginseng-Schisandra Decoction treatment of RRTI than previously thought. The results may be related to the single drug quantity and chemical composition. In regard to the compounds, the top three compounds in degree were MOL000098-quercetin, MOL000422-kaempferol, and MOL003896-isoflavone. Quercetin, kaempferol, and isoflavones are all flavonoids. Modern pharmacology has confirmed that quercetin has anti-inflammatory, antibacterial, antioxidant, antiviral, antihistamine, vasodilation, regulation of immune function, and other effects (16), and can interact with viral surface proteins to block viral adhesion (17). Kaempferol has anti-infection, anti-inflammatory, anti-cancer, and other effects (18). Isoflavones have significant antioxidant activity and can scavenge free radicals (19). These findings indicate that Modified Ginseng-Schisandra Decoction may exert a 
synergistic effect through its main components, which jointly treat RRTI. In terms of the target, the top five degree values were PTGS2, ESR1, AR, PPARG, and NOS2. PTGS2/COX-2 is a key inducible enzyme that catalyzes prostaglandin synthesis in inflammation, which is enhanced in inflammatory stimulation, tissue damage, and other situations, and participates in the inflammatory response (20). Modern pharmacology has further confirmed that the antiinflammatory activity of quercetin (21) and kaempferol (22) can act by mediating the expression of COX-2. Kaempferol could inhibit COX-2 expression in MCF-7 cell (23). Kaempferol-3-O-rutinoside inhibits macrophages from secreting inflammatory factors, such as TNF, like IL-6, TNF- $\alpha$, COX-2 and iNOS (24). Quercetin reduces COX-2 and MPO expression in intestine ischemia reperfusion injury (25). The NF- $\kappa \mathrm{B}$ pathway is inhibited by Quercetin (26). Isoflavone could also inflammation via regulating COX-2 and NF- $\kappa \mathrm{B}$ pathway $(27,28)$. Since Kaempferol, Quercetin and Isoflavone are the main components of Modified Ginseng-Schisandra Decoction, we speculated that the anti-inflammatory effect and treating repeated respiratory infection of Modified GinsengSchisandra Decoction might be mediated by the regulation of NF- $\mathrm{kB}$ and COX-2. However, there is no report on Modified Ginseng-Schisandra Decoction targeting COX-2 in the treatment of RRTI, and this should be investigated further. The results of the PPI network shows that STAT3, JUN, MAPK1, AKT1, MAPK3, etc. may be important target groups for the treatment of RRTI with Modified Ginseng Decoction. Among them, the STAT3 degree value was the largest, indicating that it may occupy a major position in the DDT network in this prescription. This is not consistent with PTGS2, ESR1, AR, PPARG and NOS2 being in the core position in the DDT network diagram of Modified Ginseng-Schisandra decoction. This discrepancy be due to the manner in which the Chinese herbal medicine was processed, whether it was decocted, and the specificity of target action, and thus warrants further study. STAT3 is an important node in the development of inflammation. It can regulate the biological behavior of tumor cells and immune cells by mediating extracellular signals of inflammatory mediators, and it is thus a central molecule in the process of inflammation (29). In summary, the Modified GinsengSchisandra Decoction may be used to target STAT3 to affect its normal physiological and pathological process in treating RRTI.
The results of KEGG enrichment analysis show that cancer signaling pathway, TNF signaling pathway, and HIF-1 signaling pathway were the leading signaling pathways for the treatment of RRTI by Modified GinsengSchisandra Decoction. The cancer signaling pathway contains 15 signal transduction pathway-specific marker genes related to tumor formation. Among them, the

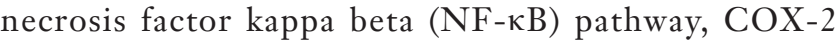
pathway, and STAT pathway may be closely related to the occurrence and development of repeated respiratory infections. The NF- $\mathrm{BB} / \mathrm{COX}-2$ pathway is an important part the important mechanism that leads to inflammatory disease, and it plays a critical role in the regulation of the body's inflammatory immune response (30). Therefore, it can be surmised that the Modified Ginseng-Schisandra Decoction may operate through signal transduction at some key nodes of the NF- $\mathrm{KB} / \mathrm{COX}-2$ pathway to effectuate RRTI treatment. Tumor necrosis factor alpha (TNF- $\alpha)$ is an important mediator and regulator of mammalian immune response in both healthy and diseased conditions. It can control the development of the immune system, cell survival signaling pathways, proliferation, and metabolic processes. Studies have confirmed that gene blockade of TNF receptor 1 (TNFR 1) expression can lead to a phenotype similar to that found in mice that lack TNF-expression, which is characterized by a lack of B cell germinal centers, resistance to liposaccharide (LPS)-mediated systemic toxicity, and increased susceptibility to bacterial infections (31). The results of this study show that the Modified GinsengSchisandra Decoction can treat RRTI through TNF signaling pathway. In conclusion, the Modified GinsengSchisandra Decoction may play a role in the treatment of RRTI through the cancer signaling pathway, TNF signaling pathway, and HIF-1 signaling pathway.

In summary, this study used the network pharmacology to conduct a preliminary theoretical investigation into the mechanism of the Modified Ginseng-Schisandra Decoction in the treatment of RRTI. The research results clarify the relationship between the main potential active ingredients and target genes of Modified Ginseng-Schisandra Decoction and the pathway of RRTI, providing new ideas for further in-depth exploration of the mechanism of action. The limited information in current databases and the lack of support of experimental results should be considered the limitations of our study. Despite this, the results can be used to direct future research, with further in-depth experimental 
investigation being needed in the future.

\section{Acknowledgments}

Funding: Shanghai Municipality's three-year action plan for further accelerating the development of Chinese medicine (2018-2020) [ZY(2018-2020)-FWTX-4023]; Shanghai Municipal Health Commission's Maternal and Child Health TCM Special Construction Project (FYJKZY-EB-2).

\section{Footnote}

Reporting Checklist: The authors have completed the MDAR reporting checklist. Available at https://dx.doi. org/10.21037/tp-21-240

Conflicts of Interest: All authors have completed the ICMJE uniform disclosure form (available at https://dx.doi. org/10.21037/tp-21-240). The authors have no conflicts of interest to declare.

Etbical Statement: The authors are accountable for all aspects of the work in ensuring that questions related to the accuracy or integrity of any part of the work are appropriately investigated and resolved. The study was conducted in accordance with the Declaration of Helsinki (as revised in 2013).

Open Access Statement: This is an Open Access article distributed in accordance with the Creative Commons Attribution-NonCommercial-NoDerivs 4.0 International License (CC BY-NC-ND 4.0), which permits the noncommercial replication and distribution of the article with the strict proviso that no changes or edits are made and the original work is properly cited (including links to both the formal publication through the relevant DOI and the license). See: https://creativecommons.org/licenses/by-nc-nd/4.0/.

\section{References}

1. Diseases S, Pediatrics CMASO. Clinical concept and management of recurrent respiratory tract infections in children (revised). Zhonghua Er Ke Za Zhi Chinese Journal of Pediatrics 2008.

2. Wang S, Chen Z, Dai Q. Guidelines for diagnosis and treatment of varicella in Chinese medicine. Journal of Pediatrics of Traditional Chinese Medicine 2011.

3. Bellanti JA. Recurrent respiratory tract infections in paediatric patients. Drugs 1997;54 Suppl 1:1-4.

4. Rudan I, Boschi-Pinto C, Biloglav Z, et al. Epidemiology and etiology of childhood pneumonia. Bull World Health Organ 2008;86:408-16.

5. Chen HZ, Hu YJ. How should we understand the recurrent respiratory tract infections? Zhonghua Er Ke Za Zhi 2008;46:83-4.

6. Li HX. Research Progress on Prevention and Treatment of Children's Recurrent Respiratory Infections With Integrated Traditional Chinese and Western Medicine. The Journal of Medical Theory and Practice 2019;32:1318-20.

7. Otolaryngology Professional Committee PS, Chinese Medical Doctor Association. Expert Consensus on Clinical Diagnosis, Treatment and Management of Repeated Upper Respiratory Tract Infections in children. Chinese Journal of Practical Pediatrics 2017;32:721-5.

8. Xu YX, Huo LL,Wu YL, et al. Randomized Controlled Trial of Two Sequential External Treatment Protocols for the Recurrent Respiratory Tract Infection in Children. Journal of Traditional Chinese Medicine 2020;v.61:64-9.

9. Cao BC, Lu YF. The Etiology, Pathogenesis and Treatment of Children With Recurrent Respiratory Tract Infection. Hubei Journal of Traditional Chinese Medicine 2014;36:71.

10. He JW, Wang YH, Sun W, et al. Analysis on professor SHI Yumin's experience in the treatment of recurrent respiratory tract infection in children and composing principle based on traditional Chinese medicine inheritance support platform(V2.5) software Journal of Pediatrics of Traditional Chinese Medicine 2020;16:23-8.

11. Li H, Li J, Guo TT. Decoction in the treatment of spleen deficiency syndrome due to recurrent respiratory infection in children: 89 cases report Shanghai. Journal of Traditional Chinese Medicine 2018;52:37-9.

12. Li H, Li J, Guo TT. Clinical Study of Using GinsengSchisandra Decoction in the Treatment of Spleen Asthenia Syndrome after Recurrent Respiratory Infection in Children. Journal of Sichuan of Traditional Chinese Medicine 2018;36:71-3.

13. Kanehisa M, Goto S. KEGG: kyoto encyclopedia of genes and genomes. Nucleic Acids Res 2000;28:27-30.

14. Zhou K, Zhang Y, Guan W, et al. Analysis of differences between ginseng and dangshen on Shengmaiyin based on integrative pharmacology of traditional Chinese medicine. Chinese Archives of Traditional Chinese Medicine 2020; Available online: http://kns.cnki.net/kcms/detail/21.1546. R.20200817.0930.038.html 
15. Li XF. Survey of research on the chemical constituents and pharmacological effects of Codonopsis Chinese Journal of Rural Medicine and Pharmacy 2014;21:83-4.

16. Xin G, Li X, Huang XW. Research progress on chemical constituents and pharmacological action of radix sileris. Jilin Journal of Chinese Medicine 2018;38:1323-5.

17. Zhang LQ, Sha F, Cui GZ, et al. Progress in pharmacological research of schisandra lignans. Journal of GuiZhou College of Traditional Chinese Medicine 2014;36:55-8.

18. Xu TF, He CG, Yang K. Network pharmacology-based study on material basis and mechanism of Qingfei Paidu Decoction against COVID-19. Natural Product Research and Development 2020;32:901-8.

19. Zang YJ, Lan T, Chu Q, et al. Progress in preparation technology and functional activity of soybean isoflavones. Journal of Food Safety \& Quality 2020;11:5964-70.

20. Desai SJ, Prickril B, Rasooly A. Mechanisms of Phytonutrient Modulation of Cyclooxygenase-2 (COX2) and Inflammation Related to Cancer. Nutr Cancer 2018;70:350-75.

21. Niu GM, Zhang FH, Yang GL, et al. Role of adenosine monophosphate activated kinase/cyclooxygenase-2 in quercetin inhibiting proliferation of K562 leukemia cells. Heilongjiang Medical Journal 2017;41:297-8.

22. Kong LX. Mechanism and pharmacokinetics of kaempferol on atherosclerosis. Chongqing Medical University 2014.

23. Zhang R, Zhang Y, Xin X, et al. Dual-Targeting Antiproliferation Hybrids Derived from 1-Deoxynojirimycin and Kaempferol Induce MCF-7 Cell Apoptosis through the Mitochondria-Mediated Pathway. J Nat Prod 2021;84:1534-43.

24. Hu WH, Dai DK, Zheng BZ, et al. The binding of

Cite this article as: Cui QK, Li H, Li Z, Li J, Song L. Study on the mechanism of the Modified Ginseng-Schisandra Decoction (MGSD) in the treatment of recurrent respiratory tract infection (RRTI) based on network pharmacology. Transl Pediatr 2021;10(6):1701-1711. doi: 10.21037/tp-21-240
kaempferol-3-O-rutinoside to vascular endothelial growth factor potentiates anti-inflammatory efficiencies in lipopolysaccharide-treated mouse macrophage RAW264.7 cells. Phytomedicine 2021;80:153400.

25. Tóth Š, Jonecová Z, Čurgali K, et al. Quercetin attenuates the ischemia reperfusion induced COX-2 and MPO expression in the small intestine mucosa. Biomed Pharmacother 2017;95:346-54.

26. Ramyaa P, Krishnaswamy R, Padma VV. Quercetin modulates OTA-induced oxidative stress and redox signalling in HepG2 cells - up regulation of $\mathrm{Nrf} 2$ expression and down regulation of NF- $\mathrm{B}$ and $\mathrm{COX}-2$. Biochim Biophys Acta 2014;1840:681-92.

27. Lau TY, Leung LK. Soya isoflavones suppress phorbol 12-myristate 13-acetate-induced COX-2 expression in MCF-7 cells. Br J Nutr 2006;96:169-76.

28. Khan AQ, Khan R, Rehman MU, et al. Soy isoflavones (daidzein \& genistein) inhibit 12-O-tetradecanoylphorbol13 -acetate (TPA)-induced cutaneous inflammation via modulation of COX-2 and NF- $\mathrm{KB}$ in Swiss albino mice. Toxicology 2012;302:266-74.

29. Yu H, Pardoll D, Jove R. STATs in cancer inflammation and immunity: a leading role for STAT3. Nat Rev Cancer 2009;9:798-809.

30. Li YY, Huang SS, Lee MM, et al. Anti-inflammatory activities of cardamonin from Alpinia katsumadai through heme oxygenase- 1 induction and inhibition of NF- $\kappa \mathrm{B}$ and MAPK signaling pathway in the carrageenan-induced paw edema. Int Immunopharmacol 2015;25:332-9.

31. Probert L. TNF and its receptors in the CNS: The essential, the desirable and the deleterious effects. Neuroscience 2015;302:2-22.

(English Language Editor: J. Gray) 